\title{
The Seismic Hazard Estimate of the Manila Trench Subduction Zone based on the Generalized Pareto Distribution
}

Jianwei Tian, Zhe Liu, Luchuan Ren *

Institute of Disaster Prevention, China Earthquake Administration, Beijing East Yanjiao, 101601

\section{基于广义帕累托分布的马尼拉海沟俯冲带地震 危险性估计}

\author{
田建伟, 刘哲, 任鲁川* \\ 中国地震局防灾科技学院, 北京东燕郊, 101601, 中国
}

\begin{abstract}
Abstraçt
The Generalized Pareto Distribution (GPD) is a distribution model of the generalized extreme value theory. The author choose the Manila trench subduction zone as a potential source area, to establish its seismic hazard estimation model based on the Generalized Pareto Distribution, by fitting magnitude data which larger than a specific threshold in a certain period. Both the return level of strong earthquake and the upper bound magnitude for that subduction zone are estimated, as well as their uncertainties are analyzed in this paper. The results show that the upper bound magnitude of the Manila Trench subduction zone is 9.2 , the magnitude return level expectation of 10 years, 50 years, 100 years and 200 years is $7.6,7.9,8.0,8.2$.respectively.
\end{abstract}

Keywords:The Generalized Pareto Distribution; threshold; upper bound magnitude; return level;

作者简介: 田建伟 (1992-), 女, 硕士研究 生, 主要从事自然灾害风险分析和 GIS 应用研

究。E-mail：jianwei_tian@126.com

*通讯作者: 任鲁川 (1958-), 男, 教授, 理 学博士, 主要从事自然灾害风险分析、海洋灾 害和地震灾害预测研究。

E-mail: renluchuan@sina.com
Manila Trench subduction zone

摘要

广义帕累托分布(GPD)是广义极值理论 的一种分布模型。本文选取马尼拉海沟俯冲带 作为潜源区, 基于广义帕累托分布, 通过对一 定时段内超过某一阈值的震级数据进行拟合, 建立该潜源区地震危险性估计模型, 估计强震 重现水平和震级上限, 并对估计结果的不确定 性进行了分析, 得到马尼拉海沟俯冲带震级上 限为 9 级, 未来 10 年、 50 年、 100 年、 200 年马尼拉海沟俯冲带的震级重现水平期望值 分别为 $7.1 、 7.6 、 7.7 、 7.9$ 。

关键词: 广义帕累托; 阈值; 震级上限; 重 现水平; 马尼拉海沟俯冲带

1. 引言

估计潜在地震源区的震级上限和未来一 定时间内的震级重现水平是进行地震危险性 分析的基础性工作。目前主要有确定性估计方 法和概率估计两种方法。确定性地震危险性分 析中, 对于有历史资料记载发生过重大破坏性 地震的地区, 通常是根据历史最大地震来确定 该区域的震级上限; 历史资料记载较少的区 域, 可通过与地质条件相似的区域进行构造类 比进行推断 $[1,2]$; 地震危险性分析概率估计 
方法由 Cornell 在 1968 年提出, 该方法最大 的优点是给出了不同震级的地震发生的可 能性且考虑了时间的不确定性[3,4], 我国现 有地震危险性分析方法在此方法上加以改 进 $[1,5]$ 。

极值理论是概率统计的一个重要分支, 其 部分理论与方法也已被引入到地震危险性分 析研究中。陈培善等利用极值理论计算分析强 震复发周期并将其应用到地震中长期预报研 究中 [6]; 高孟潭等利用极值统计分析方法对历 史地震影响烈度的分布特征进行研究, 发现在 历史地震记录较为丰富的地区能够利用极值 统计方法进行分析[7]。钱小仕等人利用广义极 值 (Generalized Extreme Valve) 模型对台湾地 区地震危险性进行分析, 得到以往最大震级超 过 7 级的地震估计发震次数与实际发震次数 基本吻合, 并对未来发震危险性进行预测 [8]; 张锟等将广义极值模型引入到潜在地震海啸 源区震级上限估计, 对琉球海沟俯冲带的地震 危险性进行分析[9]。

近年来, 国内外已有学者将广义帕累托分 布应用到地震预测研究中。Pisarenko 等人利用 广义帕累托分布对 1977-2000 年哈佛地震目录 中的 18 个地震区的浅源地震 $(0-70 \mathrm{~km})$ 地震矩 的分布进行了分析[10]; 钱小仕等利用广义帕 累托分布对云南地区的地震活动性参数进行 分析, 通过与基于 G-R 关系进行的预测比较, 显示基于广义帕累托分布估计的结果拟合较 好 [11]。

利用广义极值理论估计潜在地震源区的 地震危险性首先要通过选取一定时间步长, 对 震级数据按时间顺序进行区间等分, 然后选取 各区间的最大值组成最大震级样本, 对其进行 广义极值分布拟合。但由于震级数据具有波动 性, 在进行各区间最大震级选取时会造成极值 数据的遗漏, 且步长的选取对最后拟合的样本 构成也可能会造成一定影响, 进而影响估计结 果, 而广义帕累托模型是通过选取某一较大的 阈值, 对所有超过阈值的数据进行极值分布拟 合，有效的使用了极端数据 $[12,13]$ 。

2006 年, 经美国地质调查局海啸源研究 组研究推断, 南中国海存在 3 个风险较高的引 发海啸的潜源区, 分别为马尼拉俯冲带、琉球
冲带是南中国海最具危险性的潜在海啸源[14], 一旦发生灾害性地震海啸, 会对我国东南沿海 城市及台湾地区造成重大损失。Philip 等认为 在过去的 100 年时间内, 马尼拉海沟潜在地震 海啸源区没有记录到超过 7.6 级的地震, 意味 着未来有可能发生更大的震级的地震 [15]; Kusnowidjaja等通过分析地震数据和大地测量 数据建立地震破裂模型, 认为马尼拉海沟具备 发生 9 级地震的危险性, 以 9 级地震为例对其 进行海啸数值模拟, 结果表明该区域发生 9 级 地震将会对菲律宾、中国南部、越南等地沿海 地区将面临遭遇毁灭性海啸的风险[16], 但文 中对于马尼拉海沟地区能够发生震级只是提 出了一种假设。本文将利用广义帕累托分布, 估计马尼拉海沟潜在海啸源区地震危险性, 得 到潜源区的震级上限和一定时间内的震级重 现水平期望, 并对其不确定性进行分析, 给出 特定置信水平下的置信区间。

\section{2. 广义帕累托分布}

\section{1. 广义帕累托分布原理简介}

设 $X_{1}, X_{2} \cdots X_{n}$ 为独立同分布的随机变 量, 分布函数为 $F(x)$, 对于自然数 $\mathrm{n}$, 令 $M_{n}=\max \left\{X_{1}, X_{2}, \cdots X_{n}\right\}$, 若存在 $\left\{a_{n}>0, b_{n} \in R\right\}$ 和非退化函数 $G(x)$, 使 $P_{r}\left\{M_{n}\right\} \approx G(x)$, 则称 $G(x)$ 为极值分布, 极值分布存在三种形式[17], 其一般分布形式 为:

$G(x)=\exp \left\{-\left[1+\xi\left(\frac{z-\mu}{\sigma}\right)\right]^{-1 / \xi}\right\}$

其中 $\mu$ 为位置参数, $\xi$ 为位置参数, $\sigma$ 为尺 度参数。当 $\xi=0$ 时为第 I 类 Gumbel 分布; $\xi>0$ 为第 II 类 Frechet 分布, 前两类函数均 沿右卜㫜估. 半 $\varepsilon<n$ 时 为第III米 W/aihu11 
Risk Analysis and Crisis Response in Big Data Era (RAC-16)

分布, 函数存在上限值。

选取某一固定值 $u$ 为阈值, 若 $X_{i}>u$,

则称为超阈值, 称 $y=X_{i}-u$ 为超出量, 则

$F_{u}(y)=P_{r}(X-u \leq y \mid X>u)=\frac{F(u+y)-F(u)}{1-F(u)}, y>0$

称为变量 $\mathrm{X}$ 超过阈值 $u$ 的超出量分布函数。当 选定一个较大的阈值 $u$ 时, 超出量 $\mathrm{y}$ 近似服从 广义帕累托分布:

$$
H(y)=1-\left(1+\frac{\xi y}{\widetilde{\sigma}}\right)^{-1 / \xi}, \quad\left(y>0 \text { 且 }\left(1+\frac{\xi y}{\widetilde{\sigma}}\right)>0\right)
$$

其中 $\tilde{\sigma}=\sigma+\xi(u-\mu)$, 广义帕累托分布 (GPD) 与广义极值分布 (GEV) 具有相同的 形状参数。

\section{2. 基于广义帕累托分布的潜源区地震危险 性估计}

(1) 震级上限的估计
广义帕累托分布与广义极值分布具有相 同的形状参数 $\xi$, 考虑到潜在震源区的震级上

限必定为有限值, 因此只有当 $\xi<0$ 时, 超出 量分布函数为第III类 Weibull 分布时, 才符合 震级存在上限的条件, 震级上限为:

$$
\bar{x}=u-\tilde{\sigma} / \bar{\xi}
$$

（2）震级重现水平期望的估计

准确判断重大自然灾害的发生趋势是灾 害研究的热点问题[18], 震级重现水平是指未 来一段时间内可能出现的最大震级。在震级估 计重现水平时, 根据已有的 $t$ 年 $n$ 个历史地震 数据, 地震平均每年有 $n / t$ 个观测样本, 那么 未来 $\mathrm{N}$ 年的震级重现水平期望为:

$$
\begin{aligned}
& x_{N}=u+\frac{\tilde{\sigma}}{\xi}\left[\left(N * n / t * \zeta_{u}\right)^{\xi}-1\right] \\
& \text { 令 } m=N * n / t, \quad \text { 公式(5) 可写为: } \\
& x_{N}=u+\frac{\tilde{\sigma}}{\xi}\left[\left(m * \zeta_{u}\right)^{\xi}-1\right]
\end{aligned}
$$

$$
\text { 其中 } \zeta_{u}=P_{r}(X>u) \text {, 表示超过阈值的 }
$$

样本个数与总样本的比值。

重现水平期望 $x_{N}$ 估计的方差为:

$$
\operatorname{Var}\left(x_{N}\right) \approx \nabla x_{N}^{T} V \nabla x_{N}
$$

其中 $V$ 表示 $\left(\zeta_{u}, \tilde{\sigma}, \xi\right)$ 的协方差矩阵为:

$$
\begin{aligned}
V= & {\left[\begin{array}{ccc}
\zeta_{u}\left(1-\zeta_{u}\right) / n & 0 & 0 \\
0 & v_{1,1} & v_{1,2} \\
0 & v_{2,1} & v_{2,2}
\end{array}\right] } \\
\nabla x_{N}{ }^{T}= & {\left[\frac{\partial x_{N}}{\partial \zeta_{u}}, \frac{\partial x_{N}}{\partial \tilde{\sigma}}, \frac{\partial x_{N}}{\partial \xi}\right] } \\
= & {\left[\tilde{\sigma} m^{\xi} \zeta_{u}^{\xi-1}, \xi^{-1}\left\{\left(m \zeta_{u}\right)^{\xi}-1\right\},\right.} \\
& \left.-\tilde{\sigma} \xi^{-2}\left\{\left(m \zeta_{u}\right)^{\xi}-1\right\}+\tilde{\sigma} \xi^{-1}\left(m \zeta_{u}\right)^{\xi} \log \left(m \zeta_{u}\right)\right]
\end{aligned}
$$

\section{3. 马尼拉海沟俯冲带地质构造及地震活动性} 分析

马尼拉海沟（Manila Trench）位于中国南 海中央海盆东侧, 是南海唯一的海沟, 与马尼 拉海沟断裂位置稳合, 北起巴士海峡南端, 与 台湾碰撞带相连, 向南延伸到民都洛岛西侧, 总体呈南北向分布, 西侧为南海海盆, 东部为 吕宋岛弧, 表现为向西凸的弧形 (图 1), 海 底地形复杂, 起伏较大, 平均水深为 $4800 \sim 4900 \mathrm{~m}$, 长约 $1000 \mathrm{~km}$, 是菲律宾板块和 欧亚板块的分界[19-21]。

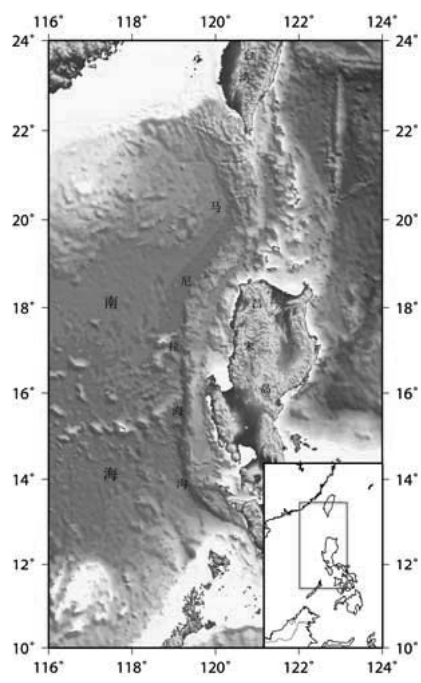

图 1 马尼拉海沟海底地形图 
Risk Analysis and Crisis Response in Big Data Era (RAC-16)

从地震活动性角度分析, 南海 6 级以上地 震几乎都集中在马尼拉海沟与吕宋海槽, 马尼 拉海沟俯冲带主要为密集的浅源地震。据美国 地质调查局地震数据资料显示, 马尼拉海沟潜 在地震海啸源区 $\left(12^{\circ}-22^{\circ} \mathrm{E}, 118^{\circ}-122^{\circ} \mathrm{N}\right)$ 以 浅源地震为主, 自 1900 年至 2015 年共发生 6 级以上地震 102 次, 6.5 级以上 37 次, 7 级以 上地震 15 次。马尼拉海沟海底地形复杂, 起 伏较大, 地震活动频繁, 被认为是一条正在活 动的、具有特殊意义的重要汇聚边缘[22,23], 且该地区一旦发生破坏性地震海啸, 将会对我 国东南沿海城市及台湾地区造成重大损失。

\section{4. 马尼拉海沟俯冲带地震危险性估计}

本文选取美国地质调查局1900-2015年地 震数据资料, 对马尼拉海沟潜在地震海啸源区 地震危险性进行分析。在利用广义帕累托分布 估计潜源区地震危险性时保证数据独立, 首先 要对地震数据进行余震的删除, 本文所采用的 是Console提出的C-S法[24], 然后根据剔除余 震后的震级数据绘制平均超出量分布函数图 （图2）进行阈值 $u$ 的选取，一般根据数据平 均超出量函数是否具有线性[12], 由图3可以看 出, 在[4.6,5.1]、[5.7,6.2]以及大于 6.5 时, 平均 超出量近似呈线性。为了对阈值进行最合理的 选取, 还需要综合考虑样本数据的实际情况, 我们可以通过观察在选取不同阈值时, 形状参 数 $\xi$ 和尺度参数 $\bar{\sigma}$ 估计值的变化的情况（图

3) 来进行取舍, 若國值对应的超出量近似为 广义帕累托分布, 则对于大于阈值的形状参数 和尺度参数的估计值应保持不变 [13]。同时, 也可以对各种阈值选取的情况进行分析, 得到 对应于各阈值的形状参数和尺度参数, 进而计 算潜源区的震级上限, 由于潜源区的震级上限 为有限值且小于 10 , 可以对阈值的选取做出进 一步判断。由图4分析, 当阈值选定为 5.1 时, 相应的形状参数和修正尺度参数的估计值保 持不变, 因此, 我们选取马尼拉海沟潜在海啸 源区广义帕累托分布的阈值为 5.1 。

在确定阈值后, 对超出量函数进行广义帕 累托分布拟合的情况进行诊断（图4), 如果 模型较为稳定, 诊断结果将产生 4 个图形, 分 别为为 P-P图, $\mathrm{Q}-\mathrm{Q}$ 图, 重现水平图, 密度曲
线图（见图4）。当数据符合理论假设的分布 时, P-P图 (4a) 和Q-Q图 (4b) 应近似为直线; 由于 $\xi<0$, 为第III类Weibull分布, 函数存在 上限值, 因此重现水平曲线 (图4c) 为凸曲线, 且逐渐趋于有限值; 从图 $4 \mathrm{~d}$ 可以看出, 拟合的 密度曲线和数据的直方图较为一致, 拟合情况

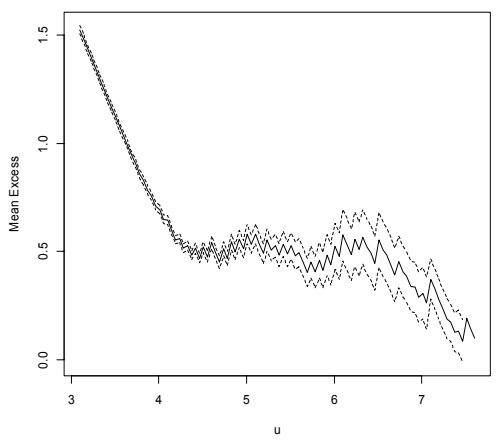

图2 平均超出量分布函数图
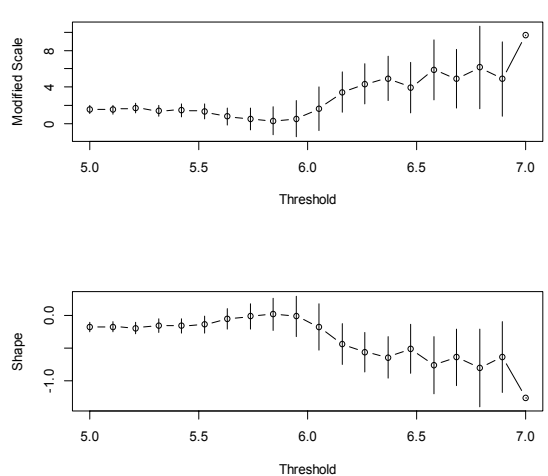

图 3 参数估计值随阈值选取的变化图

良好, 因此利用广义帕累托分布分析马尼拉海 沟潜源区的震级分布特征较为合理。

利用极大似然法估计广义帕累托分布的 参数, 得到形状参数和尺度参数的估计值:

$$
(\bar{\xi}, \widehat{\sigma})=(-0.18,0.68),
$$

其中形状参数估计的标准差为 0.04 , 其 $95 \%$ 的 置信区间为 $[-0.26,-0.10]$, 由于形状参数的取值 为负, 因此广义帕累托分布右端点为有限值, 即马尼拉海沟存在震级上限。将國值、形状参 


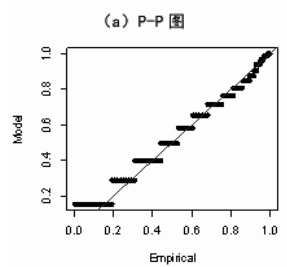

(c) 重现水平

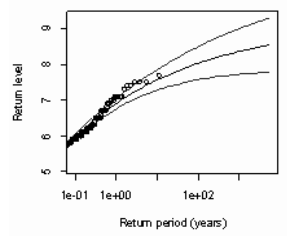

Retum period (years)

图 4 广义帕累托分布拟合诊断图

数与尺度参数估计值相应参数代入公式 (4)

得到震级上限 $M_{\text {max }}=9.0$ 。利用公式 (6) 计

算未来一定年限内的震级重现水平期望值, 得 到未来 10 年、 50 年、 100 年、 200 年的重现 水平期望值分别为 $7.1 、 7.6 、 7.7 、 7.9$ 。

由于重现水平的估计存在一定误差, 对重 现水平的误差进行分析首先要计算得到参数 估计的协方差矩阵:

$$
V=\left(\begin{array}{ccc}
3.7211 \mathrm{e}-05 & 0 & 0 \\
0 & 0.001605930 & -0.001338244 \\
0 & -0.001338244 & 0.001846948
\end{array}\right)
$$

将其代入公式 (6) 得到对应于各重现水平期 望的方差, 可得到 95\%置信度下重现水平的区 间估计, 结果见表 1 。

表 1 马尼拉海沟震级重现水平估计

\begin{tabular}{ccccc}
\hline 重现期 & $\mathbf{1 0}$ 年 & $\mathbf{5 0}$ 年 & $\mathbf{1 0 0}$ 年 & $\mathbf{2 0 0}$ 年 \\
\hline 重现水 & \multirow{2}{*}{. } & 7. & & \\
平期望 & 7.6 & 7.7 & 7.9 \\
方差 & 0.01 & 0.03 & 0.04 & 0.06 \\
$\mathbf{9 5 \%}$ 置 & {$[6.9$} & {$[7.2$} & {$[7.3$} & {$[7.4$} \\
信区间 & $7.3]$ & $7.9]$ & $8.1]$ & $8.3]$ \\
\hline
\end{tabular}

\section{5. 结论与讨论}

本文将广义帕累托分布应用于潜在地震 海啸源区的强震危险性估计中, 并选取马尼拉
海沟俯冲带进行案例研究, 得到以下主要结 论:

（1）通过对马尼拉海沟俯冲带历史地震 记录震级平均超出量分布函数进行分析, 选取 阈值为 5.1 , 计算得到震级分布的形状参数和 尺度参数取值分别为 $-0.18 、 0.68$, 推断马尼拉 海沟俯冲带震级分布为广义极值分布的第三 类即 Weibull 分布。

（2）对马尼拉海沟俯冲带强震危险性进 行分析, 得到马尼拉海沟俯冲带的震级上限估 计值为 9 级, 未来 10 年、 50 年、 100 年、 200 年马尼拉海沟俯冲带的震级重现水平期望值 分别为 $7.1 、 7.6 、 7.7 、 7.9$, 相应的 $95 \%$ 置信 度下的置信区间分别为 6.9 7.3 $]$ 、 $\left[\begin{array}{ll}7.2 & 7.9\end{array}\right]$ 、 [7.3 8.1]、[ 7.4 8.3 $]$ 。

本文得到的马尼拉海沟俯冲带震级重现 水平和震级上限估计值, 可作为震级参数选取 的参照, 供进行南中国海及沿岸地区地震海啸 危险性分析时参考。

\section{Acknowledgements}

This study was supported by National Natural Science Foundation of China (No.41276020), and the Graduate Technology Innovation Foundation of the Central Universities Fundamental Research ( ZY20160312 、 ZY20160311)

\section{致谢}

本研究得到了国家自然科学基金项目 (No.41276020)和中央高校基本科研业务费 （ZY20160312）、中央高校基本科研业务费 (ZY20160311) 的资助。

\section{参考文献}

[1]胡聿贤. 地震安全性评价技术教程. 北京: 地 震出版社, 1999.

[2]徐伟进, 高孟潭.根据截断的 G-R 模型计算 东北地震区震级上限. 地球物理学 报.2012,55(5):1710-1717.

[3]Cornell C A, Engineering seismic risk analysis. Bulletin of the Seismological Society of America, 1968,58(5):1583-1606. 
Risk Analysis and Crisis Response in Big Data Era (RAC-16)

[4]高孟潭. 地震危险性分析方法概述．国际地 震动态, 1986(11).

[5]温瑞智,任叶飞.我国地震海啸危险性分析方 法研究.世界地震工程.2007,1(23):6-11.

[6]陈培善, 林邦慧.极值理论在中长期地震预 报中的应用.地球物理学报.1973,16:6-24.

[7]高孟潭,贾素娟.极值理论在工程地震中的应 用.地震学报.1988,10(3):317-326。

[8]钱小仕;王福昌;曹桂荣;任晴晴.广义极值分 布在地震危险性分析中的应用. 地震研 究.2012,35(1):73-78

[9]张锟, 任鲁川, 田建伟, 刘哲. 基于广义极 值理论的潜在地震海啸源震级上限估计一 以琉球海沟俯冲带为例 [C]. 第十七届中国 海洋 (岸) 工程学术讨论会论文集(下). 2015,1089-1094.

[10]Pisarenko V F. Sornette D. Characterization of frequency of extreme earthquake events by the generalized pareto distribution.Pure Appl Geophys.2003:160(12):2343-2364.

[11]钱小仕, 王福昌, 盛书中.基于广义帕累托 分布的地震震级尾部分布特征分析. 地震学 报.2013,35(3):341-350.

[12]Coles S. An Introduction to Statistical Modeling of Extreme Values.A World Book Inc. 2001.

[13]史道济. 实用极值统计方法. 天津科学技术 出版社, 2006.

[14]USGS Tsunami Sources Workshop 2006: Great earthquake tsunami sources: Empiricism \&Beyond, April 21-22, 2006.

[15]Philip L.-F.Liu, Xiaoming Wang, Andrew J.Salisbury. Tsunami hazard and early warning system in South China Sea.Journal of Asian Earth Sciences. 36(2009):2-12.

[16]Kusnowidjaja Megawati, Felicia Shaw,Kerry Sieh et al. Tsunami hazard from the subduction megathrust of the South China Sea:Part I.Source characterization and the resulting tsunami. Journal of Asian Earth Sciences.36(2009):13-20

[17]Fisher $r$, Tippett L H. Limiting forms of the frequency distributions of the largest or smallest member of a sample. Proc Camb
Phil Soc,1928,24(2):180-190.

[18]Junping Yan, Shuangshuang Li, Jing Bai, Xinyan Liu. The Spatial Symmetry Axis of Earthquake Hazard in China. Journal of Risk Analysis and Crisis Response,2013, 3(1): 59-64.

[19]阮爱国,李家彪,黎明碧,等.马尼拉俯冲带的 地震学特征 [C].海底科学战略研讨会论文 集,杭州, 2003:78-85.

[20]朱俊江,丘学林,詹文欢,等.南海东部海沟的 震源机制解及其构造意义. 地震学报. 2005, 27(3):260-268.

[21]李家彪，金翔龙，阮爱国，等.马尼拉海沟 增生楔中段的挤入构造.科学通报，2004， 49(10): 1000-1008.

[22]何廉声.南海新生代岩石圈板块的演化和 沉积分布的某些特征. 海洋地质研究, 1982,2(1):16-23.

[23] 李卢玲.南海的形成与邻区构造关系.海洋 地质与第四纪地质，1985,5(1):71-82

[24]陈凌, 刘杰, 陈颗, 陈龙生.1998, 地震活动 性分析中余震的删除. 地球物理学报,41(增 刊): 244-252. 